\title{
INFLUENCE OF FLOW ON PUFF CHARACTERISTICS
}

\author{
HANA CHALOUPECKÁ ${ }^{1,2}$, ZBYNĚK JAŇOUR $^{1}$, RADKA KELLNEROVÁ ${ }^{1} \&$ KLÁRA JURČÁKOVÁ ${ }^{1}$ \\ ${ }^{1}$ Institute of Thermomechanics, Czech Republic \\ ${ }^{2}$ Charles University in Prague, Czech Republic
}

\begin{abstract}
The purpose of the study is to examine how vortices in flow influence dispersion of short-duration gas leakage (e.g. toxic gas leakage from chemical plants). A scale model of an idealized urban canopy typical for European cities was utilized. The model was composed of closed courtyards with pitched roofs placed equidistantly from each other. An approach flow angle to the model was parallel with the shorter side of the courtyards. Flow on the model was measured by Laser Doppler Anemometry. A short-duration ground-level point gas source was placed in the model. The gas leakage was simulated by ethane as a passive tracer. The gas was detected at various sampling positions within the model at pedestrian level by Fast Flame Ionisation Detector. To obtain statistically representative datasets, shortduration gas discharges were repeated under the same experimental setting about 400 times at each sampling place. From the datasets, puff characteristics (e.g. gas arrival time) were derived. Results of the flow measurements reveal channelling effect in a parallel street. In all transverse streets, recirculation vortices with vertical axes at corners of street intersections and circulation cells with horizontal axes can be spotted. The vortices in flow substantially influence puff characteristics. For example, on one hand the corner vortices seem to shorten the mean time at which gas arrives to the sampling place for some regions of the vortices. On the other hand, sampling positions near the centre of the vortices seem to be more isolated and gas therefore gets to them on an average later than to the neighbouring sampling positions. Moreover, relatively huge mean maximum concentrations detected on an average later (measured either from the release time or the cloud arrival time) than for the neighbouring sampling positions can be found in these areas.

Keywords: short-duration gas leakage, puff, contaminant, vortex, channelling effect, arrival time, maximum concentration, peak time, ascent time.
\end{abstract}

\section{INTRODUCTION}

Short duration gas discharges (e.g. leakage during plant accidents or during transport of hazardous materials) are discharges of gas that last less than one hour. Their leakage duration falls into the turbulent part of the atmospheric spectrum [1]. A gas cloud gets into a flow field presented at this region in the moment of the release. But an instant velocity field changes from moment to moment. Therefore if the gas discharge appears a little later, the dispersion of the gas determinated by the ambient conditions could be different. One realisation of the discharge represents only one from many possible dispersion scenarios. Hence, the discharge have to be repeated many times under the same experimental set-up to cover the possibilities how can a situation after gas leakage looks like. The situation at individual places affected by the leakage can be evaluated by statistical values of characteristics describing gas clouds called puffs. An overview of typical puff characteristics is given in [2].

The short duration discharges of gases that affect towns should be of special interest, since the research of such situations can help emergency services to estimate an emergency situation caused by leakage. Downtowns are usually composed of closed courdyards. Therefore many studies are engaged in research of flow and dispersion of contaminants in such or simplified urban areas composed of cuboids. For example, Moonen et al. [3] investigated effect of flow unsteadiness on the mean flow patterns utilizing RANS and LES simulations in a courtyard setting. Tablada et al. [4] specialised in ventilation of courtyards studied by both, a wind tunnel experiment and a numerical simulation. Kellnerová and Jaňour 
[5] studied flow instabilities in intersections in a courtyard setting utlizing wind tunnel modelling. Moreover, the same authors in [6] explored dispersion of pollution from a line source on the same model. Soulhac et al. [7] studied flow and dispersion from a continuous source in a street intersection on a simplified model composed of cubes by a numerical simulation as well as a wind tunnel experiment. But only few papers research dispersion from short-duration gas discharges in such urban areas. Harms et al. [8] compared puff characteristics at individual sampling places ascertained by a numerical and a wind tunnel study. Chaloupecká et al. [9] explored the change of puff characterisics within a street parallel with approach flow angle on a model composed of courtyards. But to the authors' knowledge none of studies is engaged in evaluation of influence of vortices in flow on puff characteristics, which is a main goal of the paper.

\section{METHODS}

\subsection{Experimental set-up}

Experiments were conducted in an open low speed tunnel specialized in boundary layer modelling. In the experiments, a boundary layer in 1:400 scale with a neutral stratification was used. The modelled boundary layer characteristics agree with the recommendations of VDI [10] for surfaces found in towns [11]. For incessant measurements of approach flow, Prandtl probe placed at the wind tunnel centreline was utilized. Into the tunnel, a model of 1:400 scale was placed. The model represents an idealised urban canopy found in downtowns in Europe. It consists of closed courtyards with pitched roofs placed equidistantly from each other (Fig. 1(a)). A ground-level point gas source was used for simulations of gas discharges. The utilized tracer gas was ethane. The duration of discharges was $1 \mathrm{~s}$ in the model scale. Flow measurements at the model were carried out by Laser Doppler Anemometry (LDA). The height of the measurements above the ground was the lowest technically achievable $(4 \mathrm{~m}$ in the reality). Positions of the sampling locations within the horizontal plane are displayed by blue filled circles in Fig. 1(b). Concentration measurements were done by a fast flame ionisation detector (FFID). The height of the measurements above the ground was approximately $2 \mathrm{~m}$ in the reality, which agrees roughly with the pedestrian level. The placement of the sampling positions of concentration measurements is depicted by pink unfilled diamonds in Fig. 1(b).

\subsection{Variables}

Flow measurements were used for calculations of mean velocity, skewness, variance and kurtosis, whose definitions can be found in [12]. Uncertainties of these variables were calculated with help of bootstrapping method. Since velocity time series are not independent, the block version of bootstrap [13] was used. In block resampling, the overlapping blocks were utilized since they are a little more effective than non-overlapping ones according to Hall et al. [14] and wrapped the data around a circle - used so called circular version as it has better finite sample properties since all data points get sampled with equal probability [15]. The blocks length was chosen according to Politis and White [16] with Patton et al. [17] correction. They provide an algorithm for selection of the optimal block length (in the sense of minimising the mean squared error of the estimator of the long-run variance). In this way, derived uncertainties were used for determination of lengths of intervals with the same colour tone in Figs 3 and 4. 


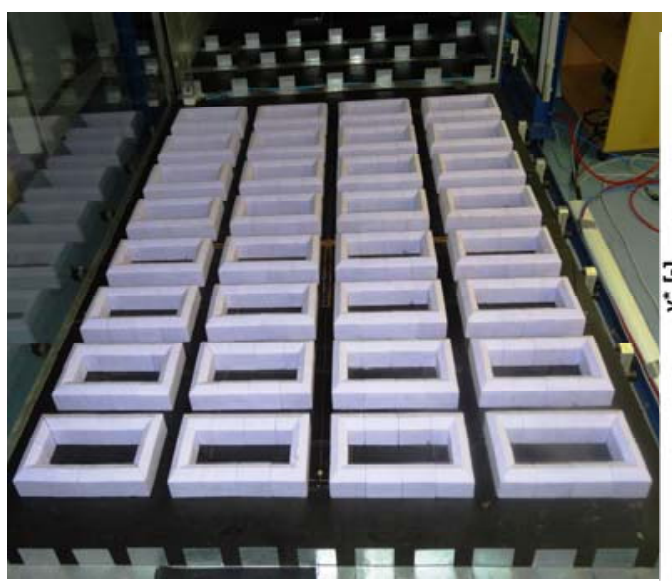

(a)

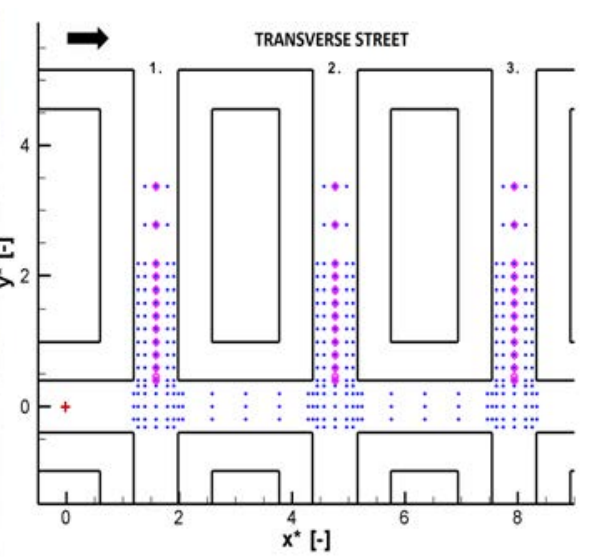

(b)

Figure 1: Model. (a) model placed in wind tunnel; (b) investigated measurement section (black arrow approach flow angle, red cross place of source, blue circles LDA positions, pink diamonds FFID positions of measurements).

The results of concentration measurements and coordinates were transformed into dimensionless forms. For the coordinates, the definition is as follows:

$$
x^{*}=\frac{x}{H}, y^{*}=\frac{y}{H} \text {. }
$$

Concentration time series were transferred into a dimensionless form by the relation:

$$
C^{*}=\frac{C U_{r e f} H^{2}}{Q} \text {. }
$$

This relation is valid for a point source [10]. The tests of this dimensionless concentration values independence on Reynolds number and on source intensity were conducted to set appropriate experimental conditions. The correctness of the time scaling was checked as well. Time was transformed to the dimensionless form utilizing the relation:

$$
t^{*}=\frac{t U_{r e f}}{H} \text {. }
$$

In these relations, $U_{\text {ref }}$ stands for the reference speed (which corresponds to the middle height of the wind tunnel), $\mathrm{H}$ the characteristic height (the height of the modelled houses $63 \mathrm{~mm}$ ) and $\mathrm{Q}$ the source intensity.

The concentration time series were analysed to obtain puff characteristics. In the paper, arrival time of puff (at) is used. It is defined utilizing threshold method with help of the knowledge of residual concentrations. This method is described in detail in [18]. Another utilized puff characteristic is a maximum concentration (pcmax). Moreover, it is explored when the maximum concentration is detected. If time is measured from the gas release, the variable is called peak time (pt). If time is measured from the arrival time, the variable is called ascent time (asct). Each concentration measurement was repeated about 400 times to obtain statistically representative datasets, what means that it is about 400 values for each puff characteristic and place. Uncertainty of the statistical values of puff characteristics (e.g., 
Table 1: Uncertainties of statistical values of puff characteristics (means, 5\%, 50\% and $95 \%$ quantiles).

\begin{tabular}{|l|l|l|l|l|}
\hline \multirow{2}{*}{$\begin{array}{l}\text { Puff } \\
\text { characteristic }\end{array}$} & \multicolumn{4}{|l|}{ Uncertainties of statistical values from ensembles } \\
\cline { 2 - 5 } & Mean & Q05 & Q50 & Q95 \\
\hline at* $[-]$ & $6 \%$ & $13 \%$ & $7 \%$ & $14 \%$ \\
\hline pcmax $^{*}[-]$ & $10 \%$ & $21 \%$ & $10 \%$ & $14 \%$ \\
\hline pt* $\left.^{*}-\right]$ & $5 \%$ & $11 \%$ & $6 \%$ & $6 \%$ \\
\hline asct* $[-]$ & $8 \%$ & $32 \%$ & $11 \%$ & $8 \%$ \\
\hline
\end{tabular}

mean arrival time) were calculated with help of bootstrapping. Percentile Bootstrap Confidence Intervals based on 10,000 bootstrap samples [13] was utilized. Typical values of uncertainties of the statistical values of puff characteristics used in the article are written in Table 1.

\section{RESULTS AND DISCUSSION}

\subsection{Flow}

Fig. 2 shows a horizontal plane of flow in investigated regions of model at height a little higher than that of the pedestrian level ( $4 \mathrm{~m}$ in the reality). Flow in a parallel street runs parallel with the approach flow direction. Within intersections, most flow continues in the same direction. According to Moonen et al. [3], the amount of the air that leaves the intersection in the approach flow direction constitutes $95 \%$ of the amount of the air entering it. At corners of the parallel street within intersections by leeward buildings flow bends into transverse streets formatting a recirculation vortex. The structure of this vortex with about a vertical axis [19] is determined by the conditions at the entrance of the intersection [7]. The closed circulation seems to occupy most of the entrance to the transverse street (more than $75 \%$ ) and is presented till about $11 / 4 \mathrm{H}$ distance from the entrance into the transverse

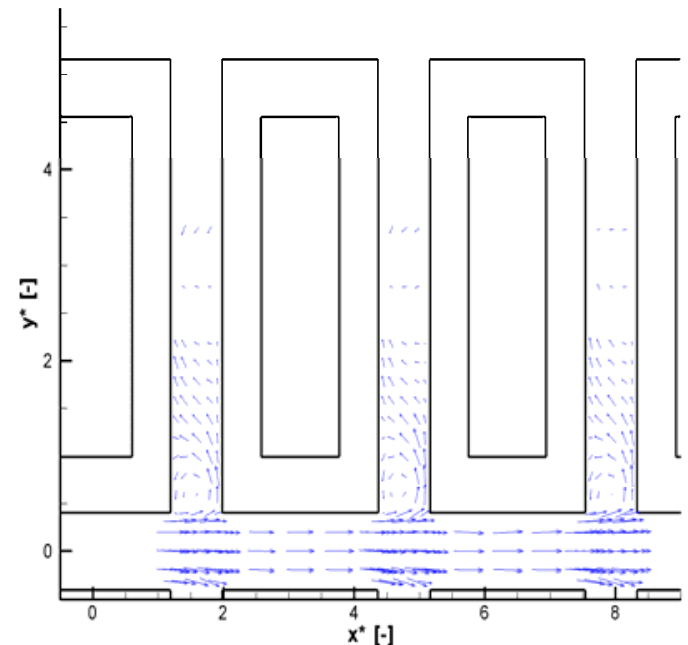

(a)

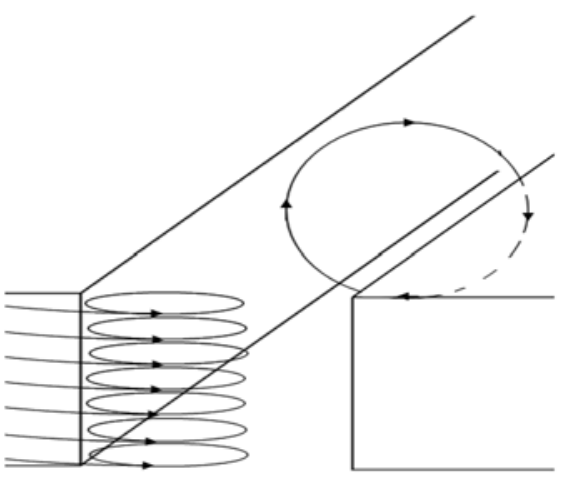

(b)

Figure 2: Flow in investigated regions. (a) Horizontal flow; (b) Simplified scheme of corner vortex and vortex with horizontal axis. 
street. The centrum of this vortex is located a little closer to the leeward building than the windward building and at a distance of about $3 / 4 \mathrm{H}$ from the entrance of the intersection. This vortex constantly slowly lifts pollutants upwards and therefore represents a relatively stable but slow ventilation of the built-up environment [5]. Far from the intersection in the transverse street, it should be expected flow resemble to the one found in the infinitely long street [7]. The flow in such development consists of a recirculating cell driven by an external flow with downward flow on the downwind face of the cavity, and an upward flow on the upwind face [19]. This structure seems to be presented at around a half of width of the transverse street since the measured vectors of horizontal wind are nearly parallel with approach flow and of opposite direction. In all along the transverse street the flow should exhibit a helicoidal structure [7].

Fig. 3 depicts horizontal flow skewness (U skew is skewness of the flow component parallel with approach flow, V skew perpendicular to it). Looking at the graph of U skewness, it can be seen rather positive skewness in the parallel street and in the transverse streets just near intersections (especially at positions within a part of a recirculation vortex closer to the intersection). That means that positive acceleration of velocity of the longitudinal component can be found often during time in these regions. Within the parallel street, this tendency is associated with channelling effect [20]. The channelling effect causes a tendency of routing air through the parallel street and accelerates flow in it. Inside the recirculation vortex, the positive skewness causes speeding up of the circulation. On the contrary, negative skewness can be found further from intersections in transverse streets (especially in the part of the street closer to the windward building) and at a part of the recirculation vortex further from the intersection. In the vortex, this causes speeding up of flow in opposite direction than that of the approach flow and enables flow to get near the leeward building to be able to close the circulation. Relatively huge negative skewness is presented in the part of the street closer to the windward building. This skewness decreases with decreasing of the distance from the leeward building because of its presence and helps to gradual straightening of the wind vectors into approach flow but of opposite direction and so creation of the recirculation cell found in the street canyons in regions at around a half of width of the transverse street. The map of $\mathrm{V}$ skewness reveals oval-shaped regions of more or less zero to little negative

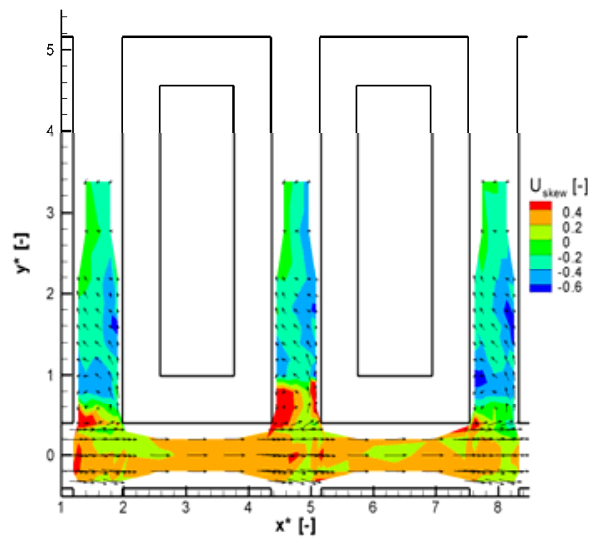

(a)

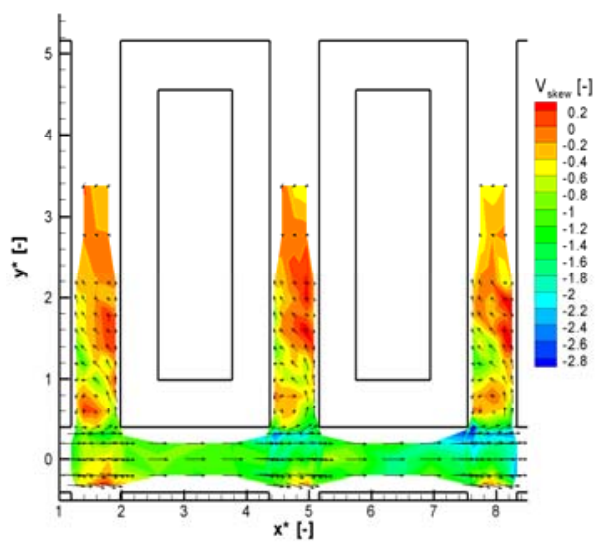

(b)

Figure 3: Skewness of flow in investigated regions. (a) Skewness of $U$ component of flow; (b) Skewness of V component of flow. 
skewness values in places where a recirculation vortex is presented. Relatively high negative values of V skewness are presented within the parallel street. Since within most parts of the parallel street flow in $\mathrm{V}$ direction is approximately zero, the negative skewness points out to the tendency to bend flow right, within intersection into the right transverse streets (seen in approach wind direction). This tendency might be caused by an unevenness of the tunnel, placement of the model parallel street longitudinal axis not exactly on the longitudinal tunnel axis and uncertainties in the model setting itself. Within intersections, it can be spotted a tendency of skewness to increase with the decreasing $\mathrm{y}^{*}$ coordinate and at the lower end of the intersection a tendency of skewness to switch to positive values. This fact causes a tendency for straightening of flow direction, which mean direction is oriented a little into transverse streets, back into approach flow angle at upper and lower boarders of intersections. This seems to be a demonstration of the routing effect in the parallel street associated with the channelling effect. Positive V skewness found in all three investigated transverse streets is situated on the windward side of the streets further from the intersections, roughly at regions of high negative $U$ skewness. A tendency to speed up flow in positive $\mathrm{V}$ direction due to the positive $\mathrm{V}$ skewness therefore in these regions seems to act against the effort of $\mathrm{U}$ skewness of straitening flow into approach flow angle.

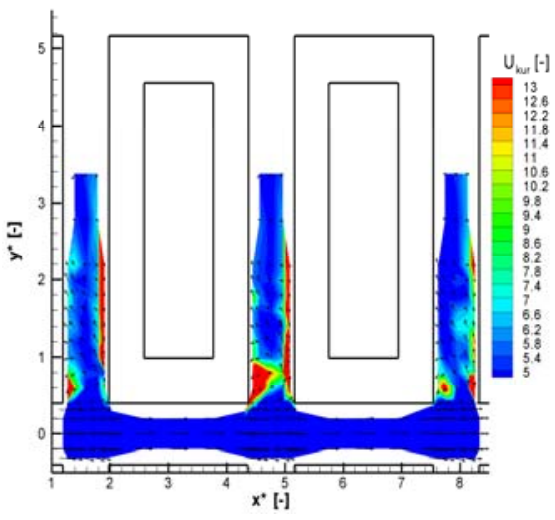

(a)

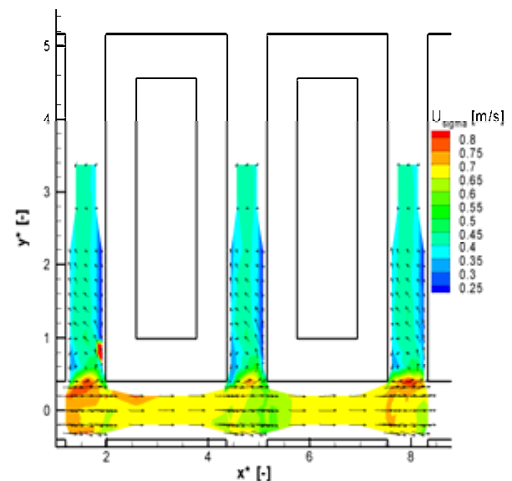

(c)

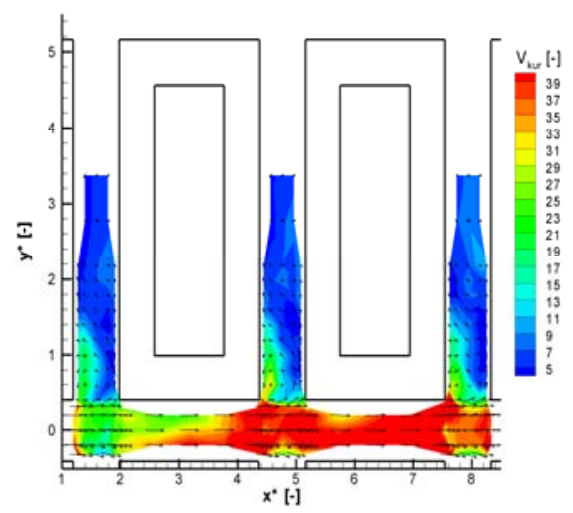

(b)

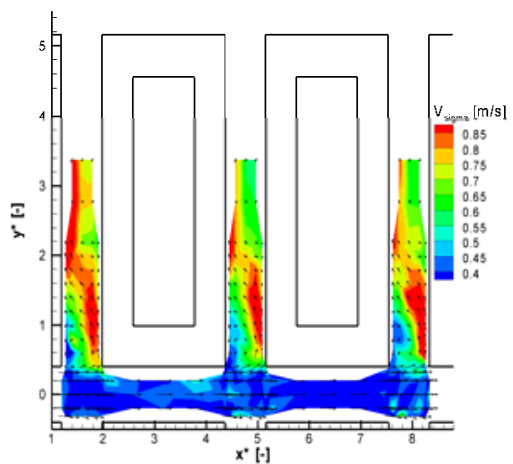

(d)

Figure 4: Kurtosis and standard deviation (root mean square of variance) in investigated regions. (a) Kurtosis of U component of flow; (b) Kurtosis of V component of flow; (c) Standard deviation of U component of flow; (d) Standard deviation of $\mathrm{V}$ component of flow. 
Fig. 4 shows kurtosis and standard deviation in investigated regions. U kurtosis (i.e., kurtosis of the flow component parallel with approach flow angle) within the parallel street is everywhere relatively small, variance of the longitudinal component of flow is rather high in the entire street. That means that distributions are flatter with heavier tails. Two types of regions with relatively high kurtosis can be spotted. The first one is located in recirculation vortices in the leeward side of the streets. That means that intermittent extreme values of the longitudinal component of flow can be often observed in these regions. The second one is situated along the windward buildings. The location of these regions roughly agrees with regions with relatively very low variances. This means that distributions have rather high peaks but because of high kurtosis longer tails meaning that in these regions dominate intermittent extreme values of the longitudinal component of flow. V kurtosis and V variance (i.e. kurtosis and variance of the flow component transverse to approach flow angle) are displayed in Fig. 4(b) and 4(d). Within the parallel street, V distributions have relatively high peaks (small variance) with long tails (high kurtosis). V velocity is approximately zero in these regions and this value usually varies only a little, but extreme events with relatively high or low values of $\mathrm{V}$ velocity can be spotted from time to time. Regions of relatively higher $\mathrm{V}$ kurtosis and lower variance jut into the transverse streets in regions of the recirculation vortices. This kurtosis roughly decreases with increase of the distance from the leeward buildings. The values of $\mathrm{V}$ velocity therefore usually do not vary a lot, but extreme intermittent events can be seen in these regions from time to time. Opposite of these regions in the transverse streets, regions with relatively high variance and low kurtosis can be found. That means that just behind the intersection in the windward side of the transverse street $\mathrm{V}$ velocity varies relatively a lot but extreme values are not spotted as often as within the recirculation vortices. Behind the recirculation vortices, these regions with relatively high variance but small kurtosis move to the leeward side of the transverse streets. These are regions close to the leeward side in which flow is gradually straightened into approach flow angle. Hence, relatively high variance of the transverse component of velocity can be spotted here.

\subsection{Short-duration gas discharges}

The results of puff characteristics are displayed into $3 \mathrm{D}$ graphs to be able to compare flow

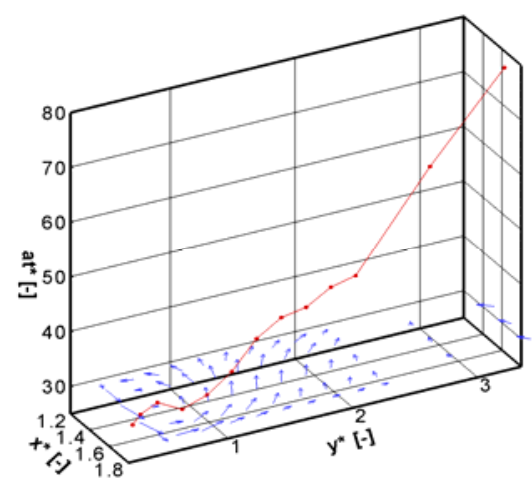

(a)

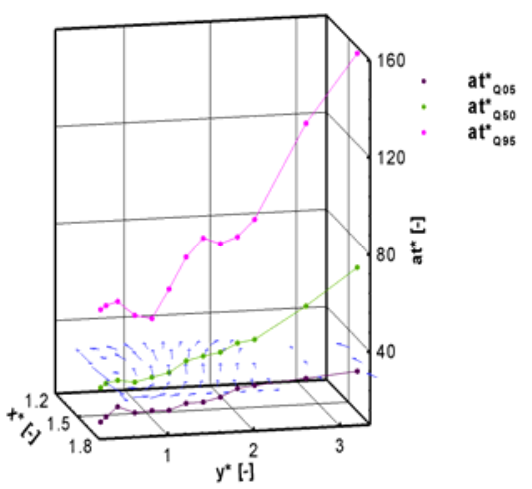

(b)

Figure 5: Arrival time (vertical axis) and flow (horizontal plane) in the transverse street closest to the source. (a) Mean arrival time with flow field; (b) Quantiles of arrival time with flow field. 
and puff characteristics easily. Mean vectors of velocity are displayed in the horizontal plane. For the places at which characteristics of gas clouds were also measured, the value of puff characteristics is plotted on the vertical axis. Results are shown on one of the investigated transverse street since more or less the same character of behaviour can be spotted in all three investigated transverse streets. This fact signifies that even when values of puff characteristics determining the character of behaviour overlap in the $95 \%$ confidence interval, the character of behaviour could be true.

Fig. 5(a) shows that mean arrival time of gas cloud increases with the growing distance from the street intersection at first, then decreases and finally again increases. The reason of such behaviour is the presence of the circulation vortex at the corner of the intersection. The vortex seems to cause speed-up of cloud arrival time at some regions. The contaminant seems to get to the places behind the centre of the vortex from the right part of the street. On the contrary, the places close to the vortex centre are more isolated by the vortex $\left(y^{*} \approx 0.6\right)$ and contaminant therefore gets to them later than to the positions more distant from the vortex centre. Influence of arrival time's quantiles by the corner vortex (Fig. 5(b)) can be also spotted. An area within the vortex, in which the quantile values of arrival time at least do not increase, is presented. Moreover, it holds that the value in places of measurements closest to the centre of the vortex $\left(y^{*} \approx 0.6\right)$ is greater than for the neighbouring places of detection within a range of quantiles values of arrival time. In the first and the third transverse street, this statement is true roughly till the $70 \%$ quantile. For the second transverse street, the statement is true only roughly till the $30 \%$ quantile. For higher quantiles, the difference of the value closest to the centre of the vortex and the neighbouring place of measurement with higher $y^{*}$ coordinate oscillates around zero. The reason of the different behaviour in the second street is probably caused by larger distance of the sampling position from the vortex centre than in the first and the third transverse street (see Fig. 2(a)). The differences in the placement of the vortex centre in the transverse streets are probably caused by uncertainties in model setting as well as the uncertainties in placement of the sampling position. Looking from the region without the closed corner vortex further from the intersection, a relatively steep increase in values for the 95th quantile in comparison with the mean can be seen. The reason is a growing distance of the sampling position from the source. Turbulent flow causes that cloud gets to the detection

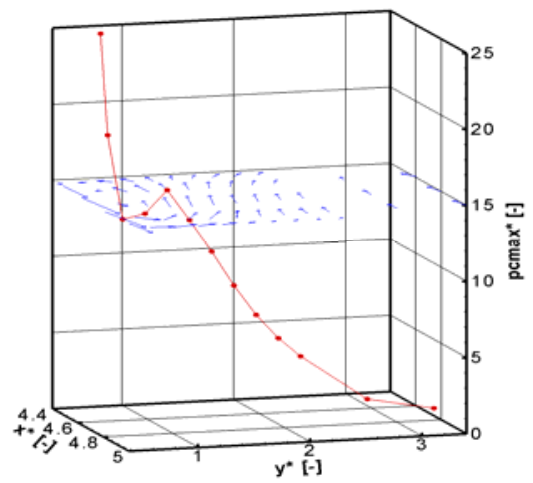

(a)

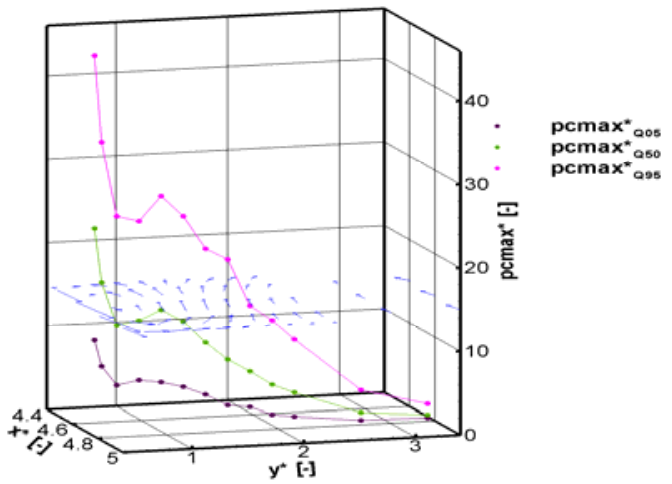

(b)

Figure 6: Maximum concentration (vertical axis) and flow (horizontal plane) in the transverse street closest to the source. (a) Mean maximum concentration with flow field; (b) Quantiles of maximum concentration with flow field. 
place in extreme runs relatively very late. This also leads to the fact that the interquartile range has a tendency to increase with the growing distance from the intersection in these regions.

Fig. 6 shows maximum concentrations in the second transverse street. The Figure reveals that the corner vortex also influences maximum concentrations. The value of maximum concentration behind the boarder of the street intersection first decreases, but then a region from the place of measurement closest to the centre of the vortex in which it can be seen an influence by the vortex begins. In this region, the value of the measured mean maximum does not decrease or measured higher value with the increasing distance from the intersection can be even spotted. Finally, the mean maximum concentration again decreases with the increasing distance from the intersection. The behaviour of the maximum concentration in the region influenced by the vortex could be explained by the following hypothesis. When the contaminant gets to the area of the vortex, it remains in the vortex and the contaminant is twisted by the vortex and therefore it can get to the observed place in the vortex again from the same, lower or upper height level of the vortex. Meanwhile another contaminant gets to the observed place. Hence, the contaminant cumulates in the vortex. This hypothesis agree with conclusions in [6] about accumulation of contaminants in corner vortices from continuous line sources. Besides the mean value of maximum concentrations, it can be also

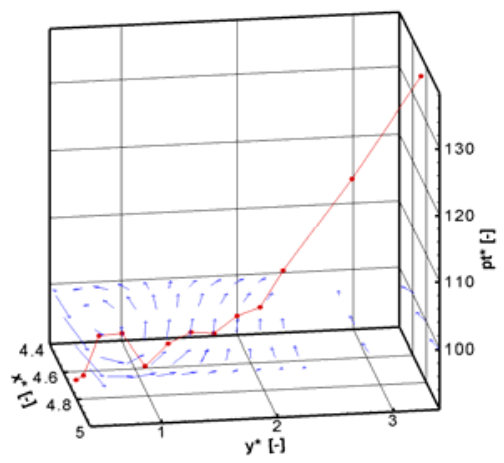

(a)

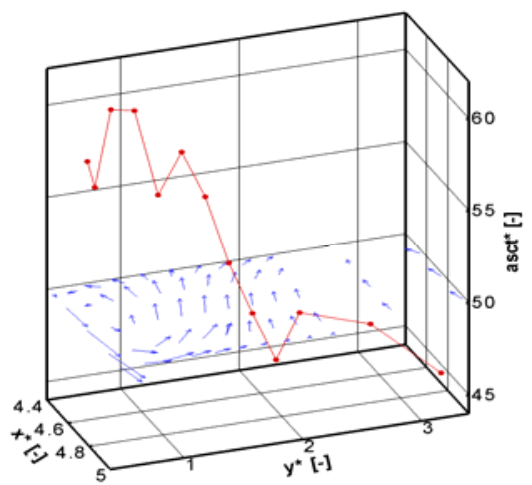

(c)

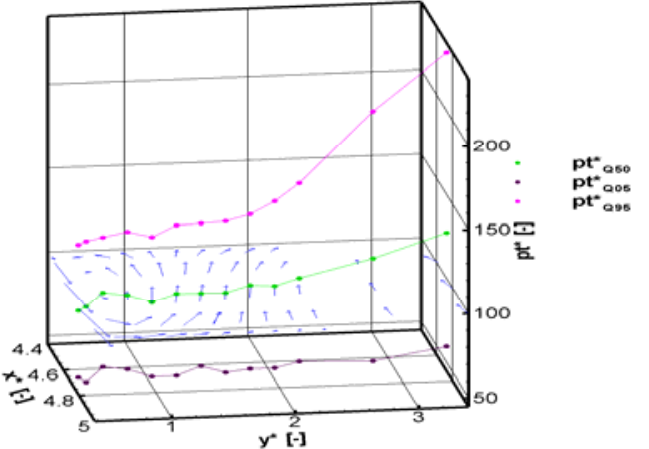

(b)

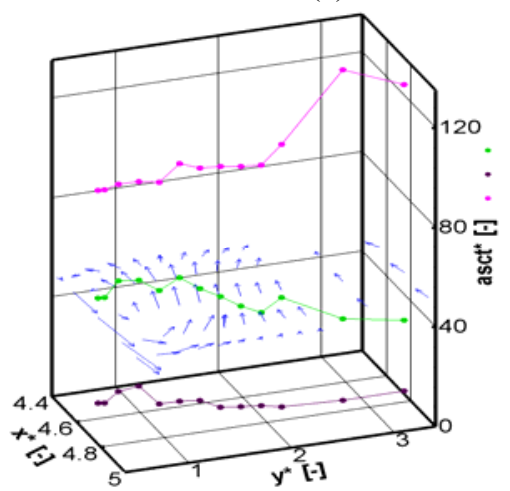

(d)

Figure 7: Time of detection of maximum concentration. (a) Mean peak time with flow field; (b) Quantiles of peak time with flow filed; (c) Mean ascent time with flow field; (d) Quantiles of ascent time with flow field. 
seen a similar character of behaviour in quantiles of maximum concentrations (Fig. 6(b)), but some changes from the first to the third transverse street can be seen. Looking from the lowest to the highest quantiles in the first transverse street, the area with almost constant concentration or even an increase in measured values with growing distance from the intersection is well expressed at first. Then it is hardly detectable for quantiles around the upper quartile and finally even higher maximum concentrations in this area than at the border of intersection can be seen. On the contrary, the flat region can be well seen at all quantiles in the second transverse street. In the third transverse street, the flat region is worse expressed for lower quantiles but it can be seen very well for higher quantiles and an increase in values within this region can be even spotted. The reason for these little changes within the streets and quantiles is probably an uncertainty of measurement of maximum concentration. These uncertainties are roughly $20 \%$ for low and high quantiles and around $10 \%$ for medians.

Fig. 7 shows time when maximum concentrations were detected. This time can be measured from the beginning of the release (peak time, Fig. 7(a) and 7(b)) or from the arrival time of gas to the detection position (ascent time, Fig. 7(c) and 7(d)). Looking from the boarder of the street intersection further into the transverse street, an increase in value of mean peak time can be spotted at first, then a decrease followed by an area with approximately slow increase in values or almost constant values. Finally, the mean value of peak time increases relatively quickly with further movement in direction from the boarder of the intersection. Median values of peak time appear more or less similar as the mean values. For high quantiles, a steeper increase in values in comparison with the mean peak time can be seen in regions further from the intersection. On the contrary, the region with increase in values far from the intersection is not almost expressed for low quantiles in the second and the third transverse street. These differences in behaviour of lower and higher quantiles lead to the tendency of the interquartile range to increase with the growing distance of the sampling position from the source. The reason is similar as for the arrival time. The more distant is the sampling position from the source, the greater differences in individual runs can be seen because of the turbulent flow. Fig. 7(c) and 7(d) shows results for ascent time. The mean value of ascent time increases within the corner vortex in comparison with the value at the boarder of the intersection, then decreases with further growing distance from the intersection and finally this decrease seems to almost stop or stops. The increase in mean values of ascent time within the recirculation vortex is in favour with the hypothesis expressed while describing maximum concentrations. The hypothesis can explain the observed character of behaviour as follows. When a central part of a cloud comes to the sampling position, a maximum concentration can be detected in regions without cumulation of contaminants. But within the vortex, the contaminant stays longer and meanwhile another contaminant gets to the position. This contaminant increases the amount of contaminant presented inside the vortex and therefore the maximum is detected later. More or less similar character of behaviour as for the mean ascent time is also presented for low quantiles of ascent time as well as the median. For high quantiles, the behaviour of ascent time changes. In the back parts of the investigated regions in the transverse streets, a tendency of values to increase with the increasing distance from the intersection can be seen.

\section{CONCLUSION}

The objective of the study was to describe how vortices in flow influence dispersion of gas clouds. Flow in urban canopy composed of rectangular closed courtyards with flow parallel with shorter side of the courtyards have been presented. In the parallel street, flow runs parallel with the approach flow. Channelling effect presented in this region causes routing of air through the street and acceleration of flow in it. Hence, positive skewness of longitudinal 
velocity in the street and increase in skewness of transverse flow component within the intersections in direction of decreasing $\mathrm{y}^{*}$ coordinate can be seen. In the transverse streets just behind the intersection, a recirculation vortex with about a vertical axis can be seen. The vortex contributes to the ventilation of the urban canopy, since it constantly slowly lifts pollution upwards [5]. Circulation inside the vortex is speeded up, as positive skewness of longitudinal component of velocity is presented mostly in parts of the vortex closer to the intersection and negative skewness of the same flow component is mostly presented in parts of the vortex further from the intersection. Relatively high kurtosis of longitudinal as well as transverse component of flow within corner vortices near the leeward side of streets points out to extreme intermittent events that can be spotted from time to time in these regions. Further from the intersection, relatively huge negative skewness of longitudinal flow component and on the contrary positive skewness of transverse flow component is presented near the windward side of streets. This fact indicates to the acceleration of flow in oblique direction. Near the half length of the transverse streets, flow runs in approach flow direction but of opposite orientation. Therefore, flow seems to resemble the one found in the infinitely long street. Such flow consists of a recirculating cell driven by an external flow with downward flow on the downwind face of the cavity and an upward flow on the upwind face [19].

Corner vortices presented in transverse streets influence puff characteristics. On one hand, the vortex seems to cause speed-up of cloud arrival time at some regions. On the other hand, the sampling positions close to the centre of the vortex centre are more isolated by the vortex and mean arrival time of gas cloud and also a range of quantiles of arrival time are greater than for the neighbouring positions of detection. The corner vortex also affects the measured maximum concentration as well as the time at which it is detected. In a region of the vortex, the value of detected mean maximum concentration does not decrease or measured higher concentration with the increasing distance from the intersection can be seen. A similar character of behaviour as for the mean maximum concentration can be seen also for quantiles of maximum concentration but with some changes from the first to the third transverse street. Moreover, these relatively huge mean maximum concentrations are detected on an average later measured either from the release or from the cloud arrival time than for the neighbouring sampling positions in these areas. The situation about the maximum concentration as well as their later detection described above could be explained by the following hypothesis. When the contaminant gets to the area of the vortex, it remains in the vortex and the contaminant is twisted by the vortex. Therefore, it can get to the observed position in the vortex again. Meanwhile another contaminant gets to the observed position. Hence, the contaminant cumulates in the vortex [6] and higher mean maximum concentration detected on an average later than for the neighbouring sampling positions can be spotted.

\section{ACKNOWLEDGEMENTS}

The authors kindly thank the Czech Science Foundation GA CR - GA15-18964S and the Institute of Thermomechanics, AS CR - RVO: 6138998 for their financial support.

\section{REFERENCES}

[1] Van der Hoven, I., Power spectrum of horizontal wind speed in the frequency range from 0.0007 to 900 cycles per hour. Journal of Meteorology, 14, pp. 160-164, 1957.

[2] Doran, J.C., Alwine, T.J., Flaherty, J.E., Clawson, K.L. \& Carter, R.D., Characteristics of dispersion in an urban environment. Atmospheric Environment, 41, pp. 3440-3452, 2007. 
[3] Moonen, P., Dorer, V. \& Carmeliet, J., Effect of flow unsteadiness on the mean wind flow pattern in an idealized urban environment. Journal of wind engineering and industrial aerodynamics, 104-106, pp. 389-396, 2012.

[4] Tablada, A., Blocken, B., Carmeliet, J., De Troyer, F. \& Verschure, H., The influence of courtyard geometry on air flow and thermal comfort: CFD and thermal comfort simulations. 22nd Conference on Passive and Low Energy Architecture, 2005.

[5] Kellnerová, R. \& Jaňour, Z., Flow instabilities within an urban intersection. Int. J. Environment and Pollution, 47, pp. 268-277, 2011.

[6] Kellnerová, R. \& Jaňour, Z., Velocity and Concentration Field in Crossroad Area. Proceedings of the 16th Annual Conference of Doctoral Students, 2007.

[7] Soulhac, L., Garbero, V., Salizzoni, P., Mejean, P. \& Perkins, R.J., Flow and dispersion in street intersections. Atmospheric Environment, 43, pp. 2981-2996, 2009.

[8] Harms, F., Leitl, B., Schatzmann, M. \& Patnaik, G., Validating LES-based flow and dispersion models. Journal of Wind Engineering and Industrial Aerodynamics, 99, pp. 289-295, 2011.

[9] Chaloupecká, H., Jaňour, Z. \& Nosek, Š., Short-term gas dispersion in idealised urban canopy in street parallel with flow direction. The European Physical Journal, 114, 2016.

[10] VDI - Verein Deutscher Ingenier, Environmental meteorology Physical modelling of flow and dispersion processes in the atmospheric boundary layer. Application of wind tunnels, VDI-Standard: VDI 3783 Blatt 12, Dusseldorf, 2000.

[11] Britter, R.E. \& Hanna, S.R., Flow and dispersion in urban areas. Annual Review of Fluid Mechanics, 35, pp. 469-496, 2003.

[12] BSA Flow Software, Version 4.10, Installation \& User's Guide, Dantec Dynamics: Denmark, 2006.

[13] Davison, A.C. \& Kinkley, D.V., Bootstrap Methods and their Application, Cambridge University Press: USA, 1999.

[14] Hall, P., Horowitz, J.L. \& Jing, B.Y., On blocking rules for the bootstrap with dependent data. Biometrika, 82(3), pp. 561-574, 1995.

[15] Politis, D.N. \& Romano, J.P., A circular block-resampling procedure for stationary data, Technical report No. 370, Stanford: California, 1991.

[16] Politis, D.N. \& White, H., Automatic Block-Length Selection for the Dependent Bootstrap. Econometric Reviews, 23(1), pp. 53-70, 2004.

[17] Patton, A., Politis, D.N. \& White, H., "Correction to "Automatic Block-Length Selection for the Dependent Bootstrap" by D. Politis and H. White'. Econometric Reviews, 28(4), pp. 372-375, 2009.

[18] Chaloupecká, H., Jaňour, Z., Mikšovský, J., Jurčáková, K. \& Kellnerová, R., Evaluation of a new method for puff arrival time as assessed through wind tunnel modelling. Process safety and environmental protection, 2017.

[19] Hunter, L.J., Johnson, G.T. \& Watson, I.D., An investigation of three dimensional characteristics of flow regimes within the urban canyon. Atmospheric Environment, 26B, pp. 425-432, 1992.

[20] Cole, T., Li, X., Eising, C. \& Princevac, M., Turbulence and channelling in a simple urban environment. 17th Symposium on Boundary Layers and Turbulence, 2006. 